\title{
A COMPARATIVE STUDY ON COLOR MODEL-BASED CONCRETE IMAGE RETRIEVAL IN DIFFERENT INVARIANT COLOR SPACES
}

\author{
Hyojoo Son \\ Chung-Ang University, Seoul, Korea \\ hjson0908@wm.cau.ac.kr \\ Changmin Kim \\ Chung-Ang University, Seoul, Korea \\ changmin@wm.cau.ac.kr \\ Changwan Kim \\ Chung-Ang University, Seoul, Korea \\ changwan@cau.ac.kr
}

\begin{abstract}
Construction progress monitoring has been recognized as one of the key elements that lead to the success of a construction project. The first requirement for effective progress monitoring is the collection and analysis of construction progress information. Through the use of image retrieval, progress information about structural components can be derived from the construction site image. In this paper, the method of color model-based, concrete image retrieval is proposed for utilization in construction progress monitoring. For effective concrete image retrieval, a comparison of concrete color models in four invariant color spaces, such as normalized rgb, HSI, $\mathrm{YC}_{b} \mathrm{C}_{\mathrm{r}}$, and CIELUV, is conducted. Then, the best color configuration and color space to model the inherent concrete color and to efficiently discriminate between concrete and other objects (or "non-concrete" objects) are determined, using Mahalanobis distance and performance measures. Experimental results show that L-U color configuration in CIELUV color space yield the optimal retrieving performance, and subsequently, the highest retrieval rate of concrete color.
\end{abstract}

KEYWORDS: color invariant, color segmentation, image processing, Mahalanobis distance, object recognition.

\section{INTRODUCTION}

In the construction industry, construction progress monitoring has been recognized as one of the key elements that lead to the success of a construction project. By performing construction progress monitoring, corrective measures and other appropriate actions can be taken in a timely manner, thereby enabling the actual performance to be as close as possible to the desired outcome, even if the construction performance deviates significantly from the original plan. However, although the progress monitoring process, which includes collecting and analyzing project progress information during the construction phase, is an important task for construction management, the construction industry currently relies heavily on manual methods. Site managers still spend a significant amount of time measuring, recording, 
analyzing, and comparing progress against the project schedule (Trucco and Kaka, 2004). This information is difficult to use to control project progress in a timely manner and is inadequate for effective construction progress monitoring (Bosche and Haas, 2007; Chae and Kano, 2007; Golparvar-Fard and Peña-Mora, 2007).

In recent years, advanced methods based on computer vision techniques that replace manual methods of analysis of the acquired images have been studied (e.g., Wu and Kim, 2004; Lukins and Trucco, 2007). These research activities illustrate the possibility of automated progress monitoring and the applicability of computer vision techniques to automated detection of objects on a construction site. However, shape feature-based image retrieval encounters some problems when the environment is complex because it is strongly affected by noise (Schiewe, 2002). Color model-based image retrieval is effective when the object has a specific color. Most types of structural components that would be tracked for the purposes of measuring progress on a construction project (e.g., steel, concrete, brick, and wood) have unique, easily identifiable colors. Therefore, the structural component can be effectively detected by defining the unique color model of the specific structural component. In order to define the unique color model of the object, it is important to identify the optimal color space that represents distinct color features of the object that are not affected by the outdoor environment.

The goal of this paper is to propose an effective color model-based, structural components retrieval method for effective progress monitoring. For effective concrete image retrieval, a comparison of concrete color models in four invariant color spaces, such as Normalized rgb, HSI, $\mathrm{YC}_{\mathrm{b}} \mathrm{C}_{\mathrm{r}}$, and CIELUV, is completed. Then, the best color configuration and color space to model the inherent concrete color that would efficiently discriminate between concrete and other objects (or "non-concrete" objects) is determined, using Mahalanobis distance and performance measures. Finally, the performance of proposed method has been validated, aiming at the concrete image acquired from the construction site, and the study's results are discussed.

\section{IMAGE RETRIEVAL USING COLOR INVARIANCE}

Color has been recognized as a salient feature for object recognition, particularly given that color is an effective cue for differentiating one object from another (Wada et al., 2005). The consistency of a single-colored object is maintained within a bounded volume in a given color space under conditions of fixed illumination (Cho et al., 2001). However, the values of colors in an RGB space, the most prevalent choice for computer graphics, are deteriorated by changes in illumination of the type often observed in outdoor environments (Bascle et al., 2006). Hence, effects caused by changes in illumination of color images acquired in an outdoor environment must be effectively handled, before color-based object recognition can be used with any degree of confidence. Moreover, since the most construction activities are performed in outdoor environments, the concrete members tend to be more exposed to light. Besides basic RGB color space, there are several other alternative color spaces, such as normalized rgb, perceptual color space (HSI), orthogonal color space $\left(\mathrm{YC}_{b} \mathrm{C}_{\mathrm{r}}\right)$, and perceptually uniform color space (CIELUV), which are developed for the purposes of illumination changes (Wesolkowski, 1999; Tsai, 2006; Kakamanu et al., 2007; Tian et al., 2009). 


\section{Normalized rgb Color Space}

To reduce the sensitivity of the distribution to the light changes, Normalized rgb color space is proposed by normalizing the three components in RGB color space. It has been observed that under certain assumptions, the differences in some object-color pixels due to lighting conditions and due to ethnicity can be greatly reduced in Normalized rgb color space. Given the above advantages, this has been a popular choice in color model-based image retrieval. Three components in Normalized rgb range from 0 to 1 . Normalized rgb is easily obtained from the RGB values by a simple normalization procedure (Moreno et al., 2001):

$$
r=\frac{R}{\sqrt{R^{2}+G^{2}+B^{2}}}, \quad g=\frac{G}{\sqrt{R^{2}+G^{2}+B^{2}}}, \quad b=\frac{B}{\sqrt{R^{2}+G^{2}+B^{2}}}
$$

\section{HSI Color Space}

The HSI color space consists of three components: hue (H), saturation (S), and intensity (I). Those are the perceptual features of color that cannot be described directly by RGB color space. The conversion of RGB to HSI is invariant to surface orientations and ambient light relative to the light source, and therefore, this makes a very good choice for color modelbased image retrieval (Kakamanu et al., 2007). The RGB variables take values of 0 to 255, and the result is scaled to a range of 0 to 360 for $\mathrm{H}$, and 0 to 1 for $\mathrm{S}$ and $\mathrm{I}$. The formulae for hue, saturation, and intensity are (Cheng et al., 2001):

$$
H=\arctan \left(\frac{\sqrt{3}(G-B)}{(R-G)+(R-B)}\right), \quad S=1-\frac{\min (R, G, B)}{I}, \quad I=\frac{(R+G+B)}{3}
$$

\section{$\mathrm{YC}_{\mathrm{b}} \mathrm{C}_{\mathrm{r}}$ Color Space}

The $\mathrm{YC}_{b} \mathrm{C}_{\mathrm{r}}$ color space is one of the orthogonal color spaces. Since it clearly separates the luminance and chrominance components, it is a favorable choice for color model-based image retrieval (Kakamanu et al., 2007). The $\mathrm{YC}_{b} \mathrm{C}_{\mathrm{r}}$ color space consists of Luminance $\mathrm{Y}$, Blue Chrominance $\mathrm{C}_{\mathrm{b}}$, and Red Chrominance $\mathrm{C}_{\mathrm{r}}$. The RGB variables take values of 0 to 255, and the result is scaled to a range of 16 to 235 for $Y$, and 16 to 240 for $C_{b}$ and $C_{r}$. The $Y_{b} C_{r}$ color space is formulated as (Shih and Cheng, 2005):

$$
\left(\begin{array}{l}
Y \\
C_{b} \\
C_{r}
\end{array}\right)=\left(\begin{array}{ccc}
65.481 & 128.553 & 24.966 \\
-39.797 & -74.203 & 112 \\
112 & -93.786 & -18.214
\end{array}\right)\left(\begin{array}{l}
R \\
G \\
B
\end{array}\right)+\left(\begin{array}{c}
16 \\
128 \\
128
\end{array}\right)
$$

\section{CIELUV Color Space}

CIELUV color space is one of the perceptually uniform color spaces. Perceptual uniformity represents how two colors differ in appearance to a human observer (Kakamanu et al., 2007). CIELUV color space consists of three components: L, U, and V. The $\mathrm{L}$ parameter corresponds roughly to luminance or brightness. The $U$ parameter primarily mimics shifts from green to red (with increasing $\mathrm{U}$ ), and the $\mathrm{V}$ parameter correlates with blue and purple colors. The $\mathrm{L}$ component has a range of 0 to 100 , the $\mathrm{U}$ component has a range of -134 to 220, and the V component, from -140 to 122. CIELUV is defined as (Cheng et al, 2001):

$$
\left(\begin{array}{l}
X \\
Y \\
Z
\end{array}\right)=\left(\begin{array}{lll}
0.607 & 0.174 & 0.200 \\
0.299 & 0.587 & 0.114 \\
0.000 & 0.066 & 1.116
\end{array}\right)\left(\begin{array}{l}
R \\
G \\
B
\end{array}\right), \quad L=116^{3} \sqrt{\frac{Y}{Y_{0}}}-16, \quad U=13 L\left(u^{\prime}-u_{0}\right), \quad V=13 L\left(v^{\prime}-v_{0}\right)
$$


When $Y / Y_{0}>0.01, Y_{0}, \mathrm{u}_{0}$, and $v_{0}$ are the values for the standard white, and

$$
u^{\prime}=\frac{4 X}{X+15 Y+3 Z}, \quad v^{\prime}=\frac{6 Y}{X+15 Y+3 Z}
$$

\section{SELECTION OF AN OPTIMIZED COLOR CONFIGURATION AND COLOR SPACES FOR CONCRETE IMAGE RETRIEVAL}

This section assesses the performance of color model-based, concrete image retrieval using color segmentation in the four invariant color spaces (Normalized rgb, HSI, $\mathrm{YC}_{b} \mathrm{C}_{\mathrm{r}}$, and CIELUV) explained in the previous section.

\section{Concrete Color Model and Color Segmentation}

Color segmentation can basically be performed using appropriate object color thresholds where object color is well modeled (Hjelmas and Low, 2001). Therefore, to determine the best color space in which to model inherent concrete color, the 990 concrete color regions of size $50 * 50$ pixels were extracted, each with different illuminations, and this resulted in 2,457,000 concrete colored pixels. Then, those pixels values were converted to four invariant color spaces. For each color space, three color configurations by means of a combination of two color component were defined. In the CIELUV color space example, the possible color configurations are L-U, U-V, and L-V. Using three color configurations in each of the four color spaces, 12 concrete color distributions were made and compared. On the basis of the distribution, the generalized distance proposed by Mahalanobis (1936) is used to define the concrete color model in each of the 12 concrete color distributions. The Mahalanobis distance has already proved its strength in object detection by considering the distribution of color values of the object and defining their color models (Majoor, 2000; Tomaz et al., 2003). In this context, the pixel color from an input image can be compared with the concrete color model by computing the Mahalanobis distance, since this distance estimates how closely the pixel color resembles the concrete color of the model (Hjelmas and Low, 2001).

Figure 1 shows an example of the variation in color segmentation that result with different Mahalanobis distances in the L-U color configuration.

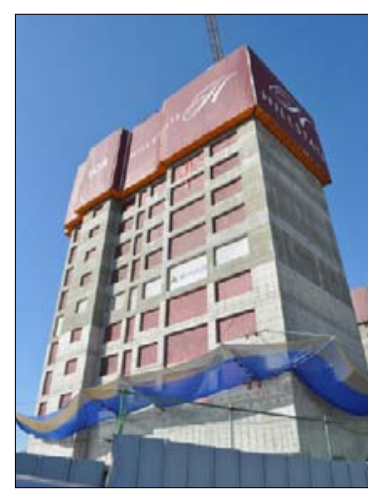

(a)

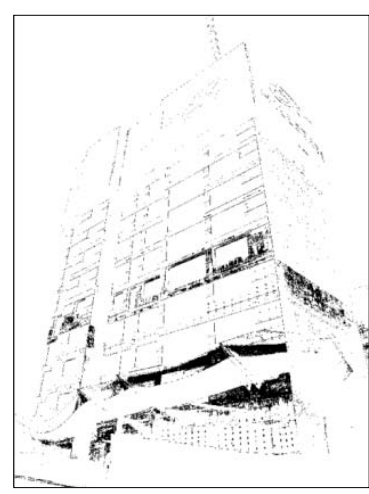

(b)

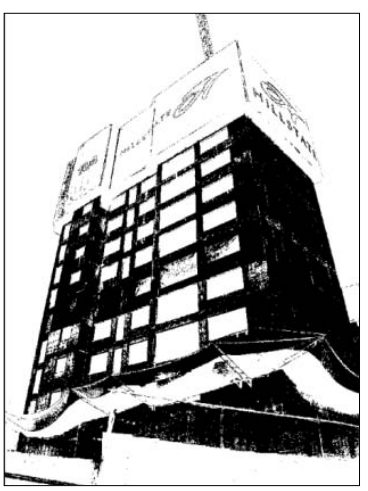

(c)

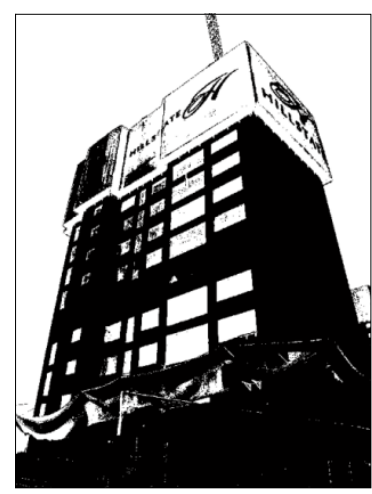

(d)

Figure 1: An Example of Color Segmentation Results with Different Mahalanobis Distances (D) in the L-U Color Configuration (a) Concrete Image, (b) $D=1$, (c) $D=5$, (d) $D=15$ 
The lower Mahalanobis distance results in an under-segmented image, while the higher Mahalanobis distance results in an over-segmented image. Therefore, analysis of the goodness of fit of the concrete distribution to the model for each of the 12 concrete color distributions, by varying the Mahalanobis distance, is required to determine the best color space to model the inherent concrete color.

\section{Comparative Evaluation Results}

The experimental tests were carried out to evaluate the concrete retrieval performance of the 12 color configurations in four color spaces, and the results are described in this section. In this study, to describe the concrete retrieval performance, the probability of error was used, as proposed by Habili et al. (2004). The probability of error as:

$$
P_{\text {error }}=P_{M}(\theta) P(w c)+P_{F}(\theta) P(w \bar{c})
$$

where $\theta$ is a threshold value, $P_{M}(\theta)$ is the probability of miss (concrete pixels detected as nonconcrete pixels), $P_{F}(\theta)$ is the probability of false alarm (non-concrete pixels detected as concrete pixels), each of $P(w c)$ and $P(w \bar{c})$ is the probabilities of the concrete and non-concrete classes in the image, and $P(w c)+P(w \bar{c})=1$.

If $P(w c)$ is unknown, the minimax test proposed by Fukunaga (1990) can be adopted to extract the threshold value. Since $P(w c)+P(w \bar{c})=1$, the previous equation can be reduced as:

$$
P_{\text {error }}=\left(P_{M}(\theta)-P_{F}(\theta)\right) P(w c)+P_{F}(\theta)
$$

To minimize $P_{\text {error }}$, threshold value should be set to make the $P_{M}(\theta)=P_{F}(\theta)$, regardless of $P(w c)$. Figure 2 shows an example of the probability of miss and false alarm of color-model based concrete image retrieval using the Mahalanobis distance in the L-U color configuration. The horizontal axis indicates the Mahalanobis distance used, and the vertical axis represents the probability of miss and false alarm. As shown in Figure 2, the probability of miss decreases along with the Mahalanobis distance, while the probability of false alarm increases. As described above, the threshold value was determined as 4.0206 when the two curves intersect.

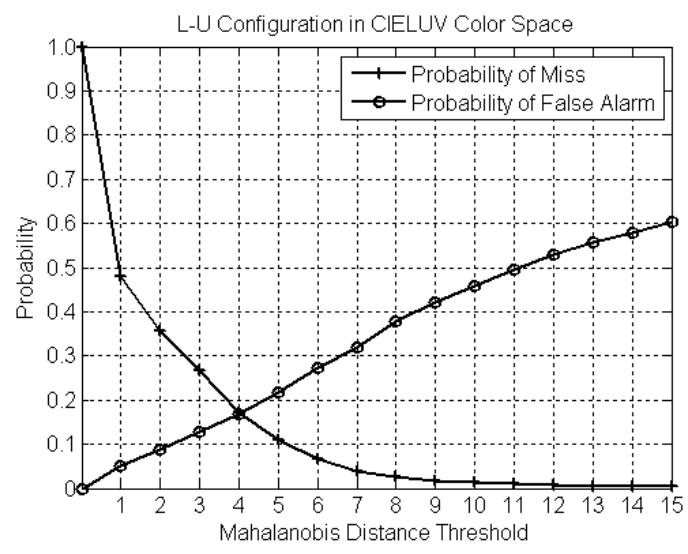

Figure 2: An Example of Probability of Miss and False Alarm in the L-U Color Configuration 
Figure 3 illustrates the images that resulted from color segmentation for Figure 1(a) with different color configurations in four color spaces, when the probability of miss and false alarm is same. From the first column to the second column, respectively, the results from 12 color configurations in Normalized rgb, HSI, YC $\mathrm{C}_{\mathrm{r}}$, CIELUV color spaces are shown. Comparing the retrieved results, it is observed that several color configurations fail to effectively distinguish concrete from non-concrete objects, as indicated in Figure 3.
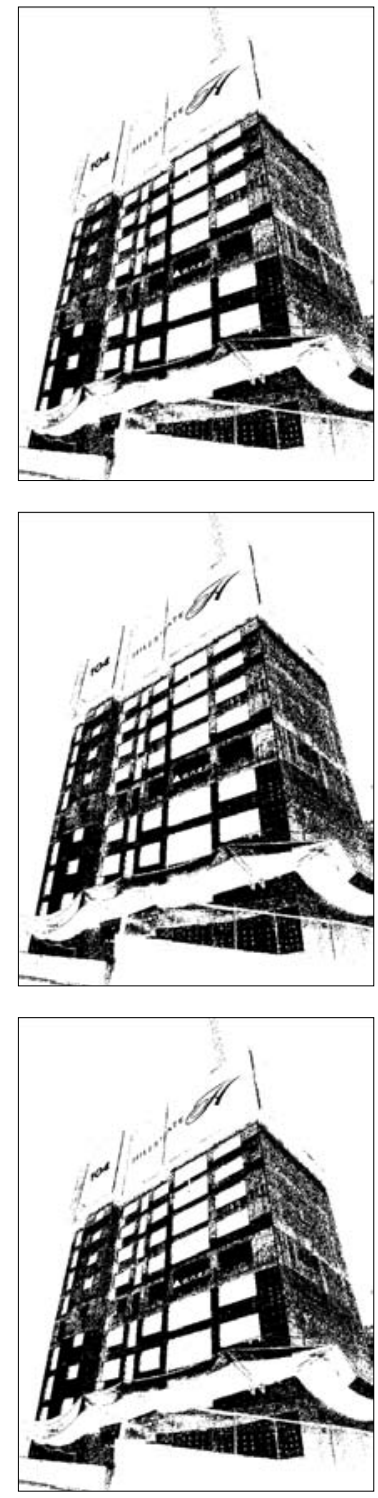

(a)
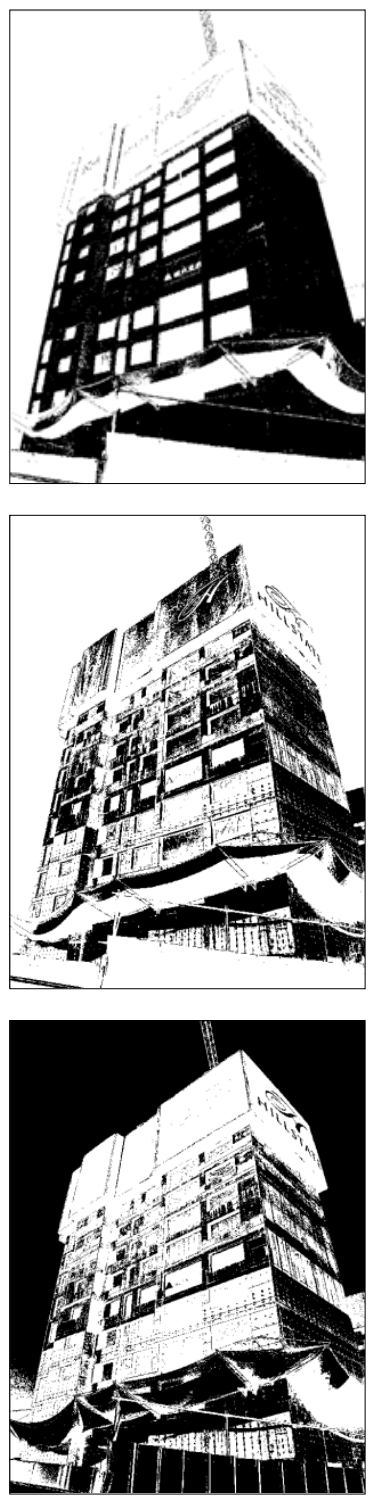

(b)
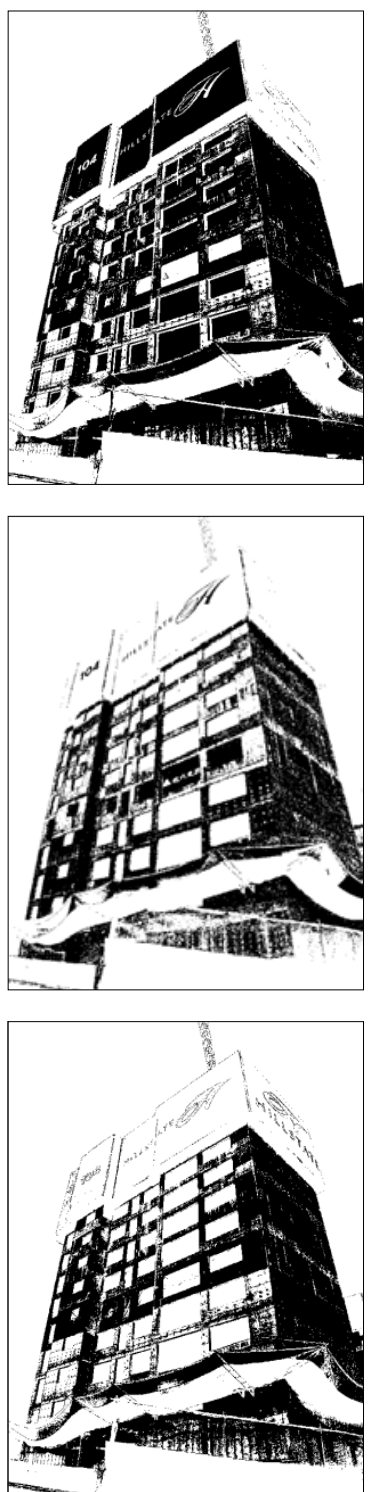

(c)
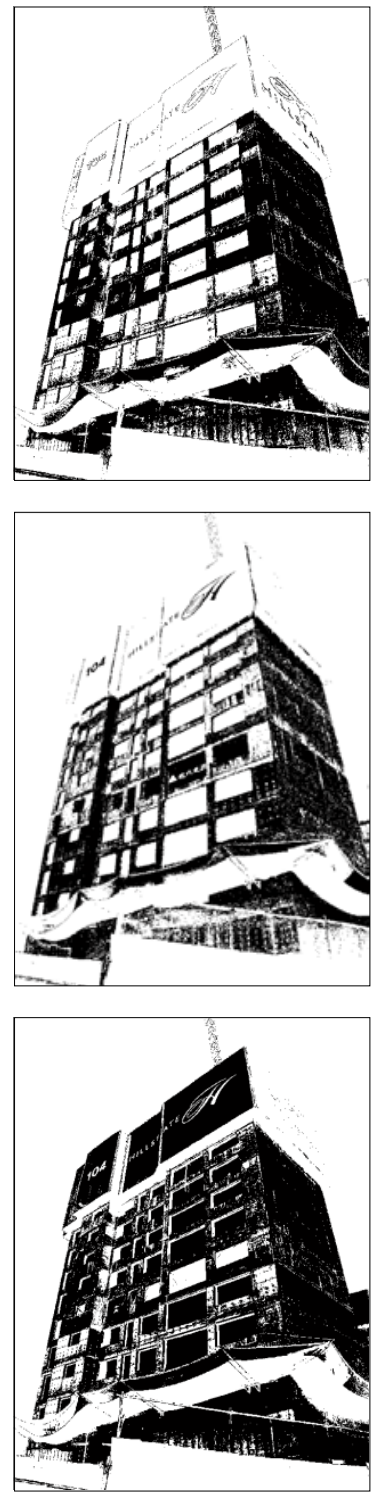

(d)

Figure 3: Results of Concrete Image Retrieval (a) r-g, g-b, r-b Color Configurations in Normalized rgb Color Space, (b) H-S, S-I, H-I Color Configurations in HSI Color Space, (c) Y-Cb, Cb-Cr, Y-Cr Color Configurations in $\mathrm{YC}_{b} \mathrm{C}_{\mathrm{r}}$ Color Space, (d) L-U, U-V, L-V Color Configurations in CIELUV Color Space

Table 1 lists the statistical verification results to highlight the most optimal color configuration and color space for concrete image retrieval. The color configurations are arranged in descending order of probability of miss and false alarm. The best performance (lowest probability of miss and false alarm) is demonstrated by the L-U color configuration in CIELUV color space, which has a probability of miss and false alarm of 0.1686 . In other 
words, the color configuration of L-U in CIELUV color space that would efficiently discriminate between concrete and other objects (or "non-concrete" objects) and produce the best retrieval results and, in this case, the Mahalanobis distance, is found to be 4.0206. The resulting image is shown in the first image in the first column in Figure 3.

Table 1: Probability of Miss and False Alarm and Mahalanobis Distances Produced on 12 Color Configurations in Four Color Spaces with Lowest Probability of Miss and False Alarm

\begin{tabular}{|c|c|c|c|c|}
\hline $\begin{array}{c}\text { Rank } \\
1\end{array}$ & $\begin{array}{l}\text { Color Space } \\
\text { CIELUV }\end{array}$ & $\begin{array}{c}\text { Color Configuration } \\
\text { L-U }\end{array}$ & $\begin{array}{l}P_{\text {error }} \\
0.1686\end{array}$ & $\begin{array}{c}\text { Mahalanobis Distance } \\
4.0206\end{array}$ \\
\hline 2 & $\mathrm{YC}_{\mathrm{b}} \mathrm{C}_{\mathrm{r}}$ & $\mathrm{Y}-\mathrm{C}_{\mathrm{r}}$ & 0.1718 & 4.1750 \\
\hline 3 & Normalized rgb & $r-g$ & 0.1755 & 2.7220 \\
\hline 4 & Normalized rgb & $r-b$ & 0.1756 & 2.8225 \\
\hline 5 & Normalized rgb & $g-b$ & 0.1779 & 2.8005 \\
\hline 6 & $\mathrm{HSI}$ & S-I & 0.1838 & 2.5834 \\
\hline 7 & CIELUV & L-V & 0.1936 & 3.7715 \\
\hline 8 & $\mathrm{YC}_{b} \mathrm{C}_{\mathrm{r}}$ & $\mathrm{C}_{\mathrm{b}}-\mathrm{C}_{\mathrm{r}}$ & 0.2053 & 3.0010 \\
\hline 9 & $\mathrm{YC}_{\mathrm{b}} \mathrm{C}_{\mathrm{r}}$ & $Y-C_{b}$ & 0.2067 & 3.7276 \\
\hline 10 & CIELUV & U-V & 0.2110 & 2.9963 \\
\hline 11 & HSI & $\mathrm{H}-\mathrm{S}$ & 0.2398 & 2.0543 \\
\hline 12 & $\mathrm{HSI}$ & $\mathrm{H}-\mathrm{I}$ & 0.3052 & 2.1025 \\
\hline
\end{tabular}

\section{CONCLUSIONS}

Progress monitoring, one of the key factors that might lead to the success of a construction project, can be conducted effectively using computer vision technology through structural components retrieval. In this study, an effective color model-based, structural components retrieval method for effective progress monitoring has been presented. A good color model must be able to discriminate between concrete and non-concrete pixels and to perform well under different illumination conditions in outdoor environments. For this reason, 12 concrete color configurations and the four color invariant color spaces were chosen for comparison and evaluation of the performance of the proposed method. The proven results from these experiments show that the proposed method is valid for concrete image retrieval, and L-U color configuration in CIELUV color space yield the best retrieving performance due to its lowest probability of miss and false alarm.

In future research, the proposed method herein will be expanded to allow for the retrieval of a number of types of structural materials, including steel, masonry, and timber, leading to a practical implementation of the method. Based on the retrieved information of structural components, it is expected that it can contribute to automated and effective construction progress monitoring. In addition, it can be utilized in the field of construction automation, such as quality control, path planning of tower cranes, and obstacle avoidance of heavy equipment at construction sites. 


\section{REFERENCES}

Bascle, B., Bernier, O., and Lemaire, V (2006) A statistical approach for learning invariants: application to image color correction and learning invariants to illumination. Proceedings of the 13th International Conference on Neural Information Processing, Hong Kong, China.

Bosche, F. and Haas, C. T (2007) Towards automated retrieval of 3D designed data in 3D sensed data. Proceedings of the 2007 ASCE International Workshop on Computing in Civil Engineering, Pittsburgh, PA.

Chae, S. and Kano, N (2007) Application of location information by stereo camera image to project progress monitoring, Proceedings of the 24th International Symposium on Automation and Robotics in Construction, Madras, India.

Cheng, H. D., Jiang, X. H., Sun, Y., and Wang, J (2001) Color image segmentation: advances and prospects. Pattern Recognition, 34, 2259-2281.

Cho, K., Jang, J., and Hong, K (2001) Adaptive skin-color filter. Pattern Recognition, 34(5), 1067-1073.

Fukunaga, K (1990) Introduction to statistical pattern recognition. Newyork: Academic

Golparvar-Fard, M. and Peña-Mora, F (2007) Application of visualization techniques for construction progress monitoring. Proceedings of the 2007 ASCE International Workshop on Computing in Civil Engineering, Pittsburgh, PA.

Habili, N., Lim, C. C., and Moini, A (2004) Segmentation of the face and hands in sign language video sequences using colour and motion cues, IEEE Transactions on Circuits and Systems for Video Technology, 14(8), 1086-1097.

Hjelmas, E. and Low, B. K (2001) Face detection: A survey. Computer Vision and Image Understanding, 83, 236-274.

Kakumanu, P., Makrogiannis, S., and Bourbakis, N (2007) A survey of skin-color modeling and detection methods. Pattern Recognition, 40(3), 1106-1122.

Lukins, T. C. and Trucco, E (2007) Towards automated visual assessment of progress in construction projects. Proceedings of the British Machine Vision Conference, Warwick, UK.

Mahalanobis, P (1936) On the generalized distance in statistics. Proceedings of National Institute of Science 2, Calcutta, India.

Moreno, F., Andrade-Cetto, J., and Sanfeliu, A (2001) Localization of human faces fusing color segmentation and depth from stereo. Proceedings of the $8^{\text {th }}$ IEEE International Conference on Emerging Technologies and Factory Automation, Antibes, France.

Schiewe, J (2002) Segmentation of high-resolution remotely sensed data - concepts, applications and problems. Proceedings of the Symposium on Geospatial Theory, Ottawa, Canada. 
Shih, F. Y. and Cheng, S (2005) Automatic seeded region growing for color image segmentation. Image and Vision Computing, 23(10), 877-886.

Tian, J., Sun, J., and Tang, Y (2009) Tricolor attenuation model for shadow detection. IEEE Translations on Image Processing, 18(10), 2355-2363.

Tomaz, F., Candeias, T., and Shahbazkia, H (2003) Improved automatic skin detection in color images, Proceedings of 12th Digital Image Computing: Techniques and Applications, Sydney, Australia.

Trucco, E. and Kaka, A. P (2004) A framework for automatic progress assessment on construction sites using computer vision. Journal of IT in Architecture, 2(2), 147-164.

Tsai, V. J. D (2006) A comparative study on shadow compensation of color aerial images in invariant color models. IEEE Transactions on Geoscience and Remote Sensing, 44(6), 16611667.

Wada, N., Tomoyuki, S., and Kanekoand, T (2005) Using color reach histogram for object search in colour and/or depth scene. Pattern Recognition, 39(5), 881-888.

Wesolkowski, S (1999) Color image edge detection and segmentation: a comparison of the vector angle and the euclidean distance color similarity measures, Master's Thesis, University of Waterloo, Ontario, Canada.

Wu, Y. and Kim, H (2004) Digital imaging in assessment of construction project progress. Proceedings of the 21st International Symposium on Automation and Robotics in Construction, Jeju, Korea. 